\title{
Was wir über die Masernimpfung wissen sollten
}

\author{
Update 2020
}

\section{What we should know about measles vaccination}

\author{
Update 2020
}

\author{
Volker Schuster \\ Universitätsklinik und Poliklinik für Kinder und Jugendliche \\ Leipzig
}

\section{ZUSAMMENFASSUNG}

Masern sind eine hochansteckende Infektionskrankheit mit einem hohen Risiko für akute und chronische Komplikationen. Die zweimalige Masern-Lebendimpfung bietet einen Schutz von bis zu $99 \%$ und könnte - sofern konsequent durchgeführt - letztlich zu einer Masernelimination führen: In Deutschland liegen die Durchimpfungsquoten immer noch deutlich unter $95 \%$. Als Folge finden sich jährliche Masernausbrüche. Aus diesem Grund wird 2020 in Deutschland eine Masern-Impfpflicht eingeführt, die nicht nur Kinder, sondern auch Erwach- sene betrifft, die im Gesundheitsdienst, in Gemeinschaftseinrichtungen (z. B. Kindergarten) oder in der Betreuung von Personen mit stark geschwächtem Immunsystem arbeiten. Die Nebenwirkungen der Masern-Lebendimpfung sind insgesamt sehr selten, können aber in wenigen Einzelfällen gravierend sein. Seltene schwere Impfkomplikationen (Enzephalitis) können u. a. bei Kindern mit Störungen der angeborenen antiviralen Immunität (Interferon-Signal-System) sowie bei schweren zellulären Immundefekten (SCID) auftreten. Säuglinge von Masern-geimpften Müttern haben niedrigere maternale anti-Masern-Antikörper als Säuglinge von Müttern, die Wildmasern durchgemacht haben. Erstere haben dadurch einen geringeren Schutz vor Wildmasern im ersten Lebensjahr.
Masern kommen ubiquitär vor. Sie sind extrem infektiös (Kontagionsindex nahe 100\%) und haben einen hohen Manifestationsindex (nahe 100\%) (RKI). Die Erkrankung zeigt einen 2-gipfligen Krankheitsverlauf mit primärem Fieber und katarrhalischen Symptomen (Konjunktivitis, Rhinitis, Halsschmerzen, Husten), lytischer Entfieberung und erneutem Fieberanstieg mit Exanthem. Häufige Komplikationen betreffen das Zentralnervensystem (Enzephalitis) und weitere Organsysteme (u. a. Myokarditis, Pneumonie) ( $\triangleright$ Tab. 1). Schlimmste und praktisch immer tödlich verlaufende Komplikation ist die subakute sklerosierende Panenzephalitis (SSPE).

Ein Charakteristikum der Masernwildinfektion - nicht aber der Masernimpfung - ist eine viral induzierte transiente Immunsuppression (, immunologische Amnesie“, u. a. Verlust von erregerspezifischen Antikörpern, Verlust von B-Gedächtniszellen), die mit einer bis zu Jahren andauernden erhöhten Empfänglichkeit gegenüber verschiedenen opportunistischen Erregern einhergeht [18, 19, 23]. Dies führt u. a. zu einer erhöhten Empfänglichkeit für bakterielle Superinfektionen (u. a. Otitis media, Bronchitis, Pneumonie und Diarrhö). Wildmasern verlaufen in 1-2/1000 Fällen tödlich. Nach überstandener Krankheit besteht lebenslange Immunität. Ein Masern-Serum-Titer von 120-300 mIU/mL gilt bei Experten häufig als Korrelat für Protektion vor Masern [3].
Masern sind keine harmlose Kinderkrankheit. Sog. „Masernparties“ (= aktive Zusammenführung nicht gegen Masern geimpfter Kinder mit Kindern, die akut an Masern erkrankt sind) erfüllen den Tatbestand der Kindeswohlgefährdung. Bei 500 betroffenen Kindern würde man hier ca. einen Todesfall „in Kauf nehmen“.

\section{Masernenzephalitis}

Zu den schweren akuten Komplikationen von Masern zählen die primäre Masern-Enzephalitis (PME) sowie die akute postinfektiöse Masern-Enzephalitis (APME). PME und APME treten während der Exanthemphase bzw. in den ersten 2 bis 4 Wochen nach akuter Masern-Erkrankung mit einer Häufigkeit von ca. einem von 1000 Masern-Fällen auf. Die Letalität der PME beträgt ca. $10 \%$. Bei 20-30\% der Betroffenen kommt es zu bleibenden Schäden. Der Verlauf der APME ist etwas günstiger [30]. Beide Komplikationen lassen sich durch frühzeitige MMR-Impfung komplett verhindern.

Die subakute sklerosierende Panenzephalitis (SSPE) ist eine chronische praktisch immer tödlich verlaufende Wildmasern-induzierte Enzephalitis. Nur geschätzte $5 \%$ der SSPE-Patienten haben eine spontane Remission [7]. Betroffen sind vor allem Kinder, die sich im Säuglingsalter angesteckt haben, nachdem ihr Nestschutz aufgebraucht war und bevor sie selbst geimpft werden konnten. Die SSPE tritt meist 1-10 Jahre nach einer Wildmaserninfektion auf. 
- Tab. 1 Komplikationen im Rahmen von Wildmasern (modifiziert nach $[1,25])$.

\begin{tabular}{|l|l|}
\hline Komplikation & $\begin{array}{l}\text { Ungefähre Häufigkeit } \\
\text { pro Fallzahl }\end{array}$ \\
\hline Gastroenteritis & $1 / 12$ \\
\hline Otitis media & $1 / 14$ \\
\hline Pneumonie & $1 / 20$ \\
\hline Krampfanfälle (mit und ohne Fieber) & $6-7 / 1000$ \\
\hline Todesfälle & $2 / 1000$ \\
\hline Primäre Masern-Enzephalitis & $1-3 / 1000$ \\
\hline Subakute sklerosierende Panenzephalitis (SSPE) & $4 / 100000$ bis $1: 1700$ \\
\hline
\end{tabular}

Nach dem derzeitigen Kenntnisstand ist sie ausschließlich mit einem genetisch veränderten Masern-Wildvirus (Mutationen in den Genen für die Masernproteine M, F und H) assoziiert [7]. Noch nie wurde bei einem an SSPE verstorbenen Patienten Masern-Impfvirus nachgewiesen. In Deutschland liegt das Risiko für eine SSPE bei Kindern mit Masern unter 5 Jahren bei $\leq 1: 1700$ [25].

Die rechtzeitige Masern-Impfung schützt vor einer SSPE (allerdings nur, wenn diese tatsächlich bereits vor einer Masern-Wildinfektion gegeben wurde). Es wird vermutet, dass Masern-geimpfte Kinder, die „trotzdem“ eine SSPE entwickeln, bereits vor der Impfung Kontakt mit dem Masernwildvirus gehabt haben müssen [7]. Es gibt weiterhin Hinweise dafür, das bestimmte genetische Prädispositionsfaktoren (angeborene Immunität: u. a. Toll-like-Rezeptoren, Zytokine) mit der SSPE assoziiert sind [7].

\section{Die Masernimpfung}

Derzeit sind in Deutschland 2 Masern-Kombinationsimpfstoffe (Dreifachimpfstoff MMR (M-M-RVaxPro ${ }^{\circledR}$, Priorix ${ }^{\circledR}$ ) oder Vierfachimpfstoff MMRV (Priorix-Tetra ${ }^{\circledR}$, ProQuad ${ }^{\circledR}$ ) verfügbar. Beide Impfstoffe sind bei 2-maliger Gabe ähnlich wirksam, immunogen und reaktogen. Beide Impfungen können problemlos zusammen mit Totimpfstoffen (z. B. 6-Fachimpfstoff DTaP-IPV-HB-Hib, Pneumokokkenund Meningokokken-Impfstoffe) verabreicht werden. Ein monovalenter Masernimpfstoff ist derzeit in Deutschland nicht verfügbar.

\section{Zeitpunkt der Masernimpfung}

Die Ständige Impfkommission (STIKO) empfielt, die erste Masernimpfung in der Regel im Alter von 11-14 Monaten. Bis zum Ende des 2. Lebensjahres soll möglichst auch die 2. Impfung erfolgt sein. Die SIKO (Sächsische Impfkommission) orientiert sich an dem amerikanischen Impfschema (https://www.cdc.gov/mmwr/pdf/rr/rr6204.pdf) und empfiehlt derzeit die Masern-Erstimpfung ab dem 13. Lebensmonat, die Zweitimpfung um den 4. Geburtstag, frü- hestens zur U8 (46.-48. Lebensmonat), bis spätestens/ oder zur Schulaufnahmeuntersuchung (https://www. slaek.de/media/dokumente/02medien/Patienten/gesundheitsinformationen/impfen/E1_2018_Druck.pdf).

Eine Masern-Impfung ab einem Alter von 9 Monaten (in Sachsen und in den USA in Ausnahmefällen bereits ab dem 6 . Lebensmonat) kann unter Berücksichtigung der gegebenen epidemiologischen Situation insbesondere in folgenden Situationen erfolgen:

- bevorstehende Aufnahme in eine Gemeinschaftseinrichtung,

- nach möglichem Kontakt zu Masernkranken.

Sofern vor dem Alter von 11 Monaten geimpft wird, muss die 2. Impfung bereits zu Beginn des 2. Lebensjahrs erfolgen, da persistierende maternale Antikörper im 1. Lebensjahr die Impfviren neutralisieren können (https://www.rki. de/SharedDocs/FAQ/Impfen/MMR/FAQ_Liste_Masern_ Impfen.html). In diesen Fällen (bei Impfalter unter 1 Jahr) ist eine zusätzliche Masernimpfdosis im Alter von 12-15 Monaten erforderlich. Diese 2 Dosen gelten zusammen als Erstimpfung (SIKO). Die Impfung erfolgt in diesen Fällen außerhalb der Zulassung, es handelt sich also um einen Off-label-Einsatz.

\section{Mütterlich übertragener Masernschutz bei jungen Säuglingen}

Die Wirksamkeit einer Masern-Impfung vor dem 6.-9. Lebensmonat ist durch das Vorhandensein mütterlicher (neutralisierender) Antikörper und durch die „relative“ Unreife des kindlichen Immunsystems vermindert. Die alleinige passive Masern-Immunität bei Säuglingen, deren Mütter Masern durchgemacht haben, hält bis zu 6 Monate lang an, bei Säuglingen von Masern-geimpften Müttern dagegen lediglich 3 Monate [26]. Ein Masern-Antikörpertiter von 120-300 mIU/mL wird häufig als Surrogatmarker für Masernschutz angenommen.

Verbessern ließe sich die Situation durch Steigerung der Herdenimmunität (Durchimpfungsquoten von > $95 \%$ ). Dass eine zusätzliche 3. Masernimpfung bei Frauen im gebährfähigen Alter die masernspezifische passive Immunität des Neugeborenen verbessern könnte, ist unwahrscheinlich. In einer kürzlich publizierten amerikanischen Studie konnte gezeigt werden, dass eine 3. Masern-Impfung bei Erwachsenen längerfristig zu keiner Zunahme der Masern-spezifischen Immunität führt [6]. > Abb. 1 zeigt, dass von 2018-2019 in Deutschland tatsächlich zahlreiche (meist nicht geimpfte) Kinder < 1 Jahr an Masern erkrankt sind.

Besonders problematisch dabei ist, dass eine Wildmaserninfektion (z. B. via ein nicht-geimpftes Kind mit floriden Masern) im ersten Lebensmonat bis -jahr mit einem stark erhöhten Risiko für eine SSPE einhergeht. Verbessert wer- 


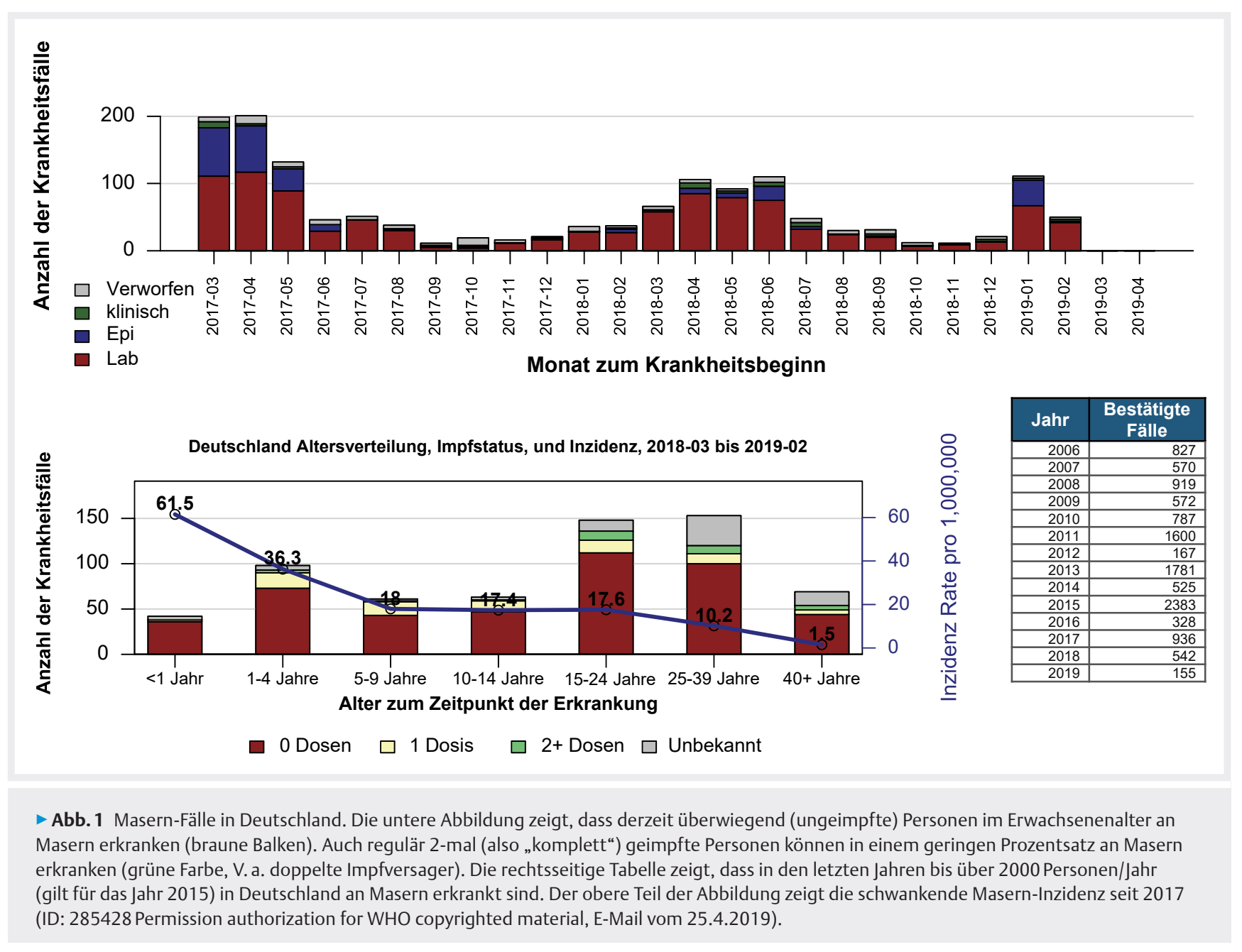

den könnte die Situation derzeit nur durch Steigerung der Masern-Herdimmunität.

\section{Wie werden die Masernimpfungen verabreicht}

Priorix-Tetra ${ }^{\circledR}$ und ProQuad ${ }^{\circledR}$ können sowohl subkutan als auch intramuskulär verabreicht werden (siehe auch Beipackzettel). Dabei konnte gezeigt werden, dass hinsichtlich Immunogenität und Reaktogenität (z. B. Schmerzen, Fieber, Lokalreaktion) kein Unterschied besteht, ob der Impfstoff subkutan oder intramuskulär gegeben wird. Die intramuskuläre Gabe wird wahrscheinlich von den meisten Patienten und Kinderärzten bevorzugt.

\section{Sind frisch Masern-geimpfte Personen für andere ansteckend?}

Die Übertragung von Masernviren (nach MMR-Impfung) vom Impfling auf empfängliche Kontaktpersonen ist bisher klinisch nicht beschrieben worden. Das betrifft natürlich auch Kinder mit sog. Impfmasern. Selbst bei Kontakt zu immunsupprimierten Personen bestehen nach derzeitigem Kenntnisstand keine Bedenken (RKI).

\section{Masern-Impfung bei Erwachsenen}

Die Ständige Impfkommission (STIKO) empfiehlt seit 2010 allen Erwachsenen, die nach 1970 geboren sind und nicht bzw. in der Kindheit nur einmal gegen Masern geimpft wurden, eine einmalige Impfung gegen Masern. Die STIKO empfiehlt die Impfung vor allem auch allen, die im Gesundheitsdienst, in Gemeinschaftseinrichtungen (z. B. Kindergarten) oder in der Betreuung von Personen mit stark geschwächtem Immunsystem arbeiten. \ Abb. 1 zeigt, dass bei Ausbrüchen überwiegend (nicht oder unzureichend geimpfte) Erwachsene an Masern erkranken.

\section{Akzidentelle Masern-Impfung in der Schwangerschaft}

Grundsätzlich sind Lebendimpfungen in der Schwangerschaft kontraindiziert. Die versehentliche Impfung mit Masern-Impfstoff in oder kurz vor einer Schwangerschaft stellt jedoch keine Indikation zur Interruptio dar (CDC-Empfehlung). Bei zahlreichen „versehentlichen“ MMR-Impfungen während bzw. kurz vor einer Schwangerschaft wurde bis heute kein erhöhtes Risiko für kongenitale Fehlbildungen festgestellt [31]. 
- Tab. 2 Komplikationen im Rahmen einer Masernimpfung (modifiziert nach [27, 31]).

\begin{tabular}{|l|l|}
\hline Komplikation & Häufigkeit \\
\hline Lokale Rötung und Schwellung an der Einstichstelle & $5 / 100$ \\
\hline Impfmasern & $5-15 / 100$ \\
\hline Gastroenteritis & 0 \\
\hline Otitis media & 0 \\
\hline Pneumonie & 0 \\
\hline Krampfanfälle & $1 / 3000$ (Fieberkrämpfe) \\
\hline Thrombozytopenie & $1 / 30000$ \\
\hline Todesfälle & 0 \\
\hline Masern-Enzephalitis & $<1 / 1000000$ \\
\hline Subakute sklerosierende Panenzephalitis (SSPE) & 0 \\
\hline
\end{tabular}

\section{Fieberkrämpfe und MMRV-Impfung}

Aufgrund eines leicht erhöhten Risikos von Fieberkrämpfen nach der Erstimpfung mit einem quadrivalenten MMRV-Kombinationsimpfstoff im Vergleich zu einer simultanen aber getrennten Gabe von MMR- und Varizellen (V)-Impfstoff wird nach dem jetzigen Kenntnisstand empfohlen, für die 1. Impfung im Alter $<5$ Jahren die getrennte MMR- und Varizellen (V)-Impfung zu verwenden (Epidemiologisches Bulletin 24. 8. 2017/Nr. 34). Die 2. Impfung gegen MMR und $\mathrm{V}$ kann dagegen mit dem MMRV-Kombinationsimpfstoff (oder simultan mit einem MMR- und V-Impfstoff) erfolgen. Diese Regelung führte allerdings bei der ersten Impfung zu einer Abnahme der Impfquote der Varizellen-Komponente (V mono oder MMRV) um 4-12\%. Bei der 2. MMRV-Impfung konnten in einer kürzlichen Studie keine Assoziationen mit Fieberkrämpfen mehr nachgewiesen werden.

Insgesamt ist es aber auch durchaus legitim, nach entsprechender Aufklärung und frühzeitiger und ausreichender Antipyrese bereits bei der 1. Impfung den MMRV-Impfstoff zu geben.

\section{Komplikationen im Rahmen der Masernimpfung}

Die MMR-Impfung wird sehr gut vertragen, Fieberkrämpfe und Lokalreaktion an der Einstichstelle sowie Impfmasern sind vergleichsweise häufig. Eine Masernimpfung-assoziierte Enzephalitis ist extrem selten (<1/1 000000$)$. Möglicherweise führt hierbei erst eine zusätzliche genetische Disposition zu dem Krankheitsbild ( Tab. 2).

\section{Masern-Impfung bei Hühnereiweiß- allergie}

Kinder mit bekannter Hühnereiweißallergie können ambulant mit Masern-Impfstoff (MMR, MMRV) geimpft wer- den. Dies belegen zahlreiche internationale Studien. Wenigstens $99 \%$ von Kindern mit nachgewiesener Hühnereiweißallergie zeigen nach einer subkutanen Dosis Masernimpfstoff keine schwere anaphylaktische Reaktion (KI=99-100\%) (14). Eine Hauttestung vor Impfung wird nicht empfohlen (5). Die sehr seltenen, beschriebenen schweren allergischen Reaktionen nach MMR-Impfung sind wahrscheinlich nicht durch Hühnereiweißallergene, sondern durch andere Komponenten des Impfstoffs verursacht (z. B. Gelatine).

Merke: Die Hühnereiweißallergie wird mittlerweile in internationalen und nationalen Leitlinien nicht mehr als Kontraindikation gegen die Masernimpfung genannt (CDC, https://www.cdc.gov/vaccines/pubs/ pinkbook/genrec.html). Ausschließlich Kinder mit klinisch bereits früher sehr schwer verlaufender, klar definitiver Hühnereiweißallergie (z. B. anaphylaktischer Schock nach Genuss von geringsten Mengen von Hühnereiweiß) sollten sicherheitshalber noch unter besonderen Schutzmaßnahmen geimpft werden (u. a. STIKO, August 2019).

\section{Besteht zwischen der Masernimpfung ein kausaler Zusammenhang mit frühkindlichem Autismus?}

Andrew Wakefield war eine der prominentesten Fake-news-Vertreter unter den Impfskeptikern, der in großem Stil u. a. die absurde These verbreitete, dass die MMR-Impfung vermehrt zu Autismus führt [29]. Die damalige Publikation wurde später aufgrund grober Fälschungen zurückgezogen. Zahlreiche internationale große Studien konnten die unhaltbaren Behauptungen wissenschaftlich immer wieder klar widerlegen [12].

\section{Vor welchen schwerwiegenden Komplikationen durch Wildmasern schützt die rechtzeitige MMR- oder MMRV-Impfung?}

Aufgrund mangelnder Durchimpfungsquoten treten in Deutschland immer wieder regionale Ausbrüche von Masern auf. Häufigste Komplikationen sind bakterielle Sekundärinfektionen (Bronchopneumonien, Otitis media und Diarrhö). Zu den Komplikationen des zentralen Nervensystems gehören die akute Masernenzephalitis mit einer Häufigkeit von 1:500-1:2000. Die Masernenzephalitis hat auch heute noch eine Letalität von $30 \%$ und eine Defektheilungsrate von ca. $20 \%$. Die Masernimpfung schützt praktisch vor allen bekannten Komplikationen der Wildmaserninfektion. 
Bei Patienten mit schwerem Immundefekt kann das Exanthem völlig fehlen (sogenannte weiße Masern). Es kann sich eine Masern-Einschlusskörper-Enzephalitis (MIBE) entwickeln, die fast immer tödlich verläuft oder mit bleibenden Schäden einhergeht. Bei immunsupprimierte Patienten ist die MMRV-Impfung daher kontraindiziert. Dieser Personenkreis kann allerdings indirekt von einer Herdimmunität profitieren, vorausgesetzt, die Durchimpfungsquote ist ausreichend hoch (möglichst $\geq 95 \%$ ).

Wie gehe ich vor bei Kindern, die aus verschiedenen Gründen nicht gegen Masern geimpft werden können oder dürfen (sog. Masern-Postexpositionsprophylaxe)

Personen mit einem erhöhten Risiko für Masernkomplikationen (Kleinkinder unter 1 Jahr, immunsupprimierte Personen) und ohne bestehende Masernimmunität können innerhalb von 6 Tagen nach Erstexposition Immunglobuline erhalten. Durch einmalige Gabe von $400 \mathrm{mg} / \mathrm{kg}$ KG eines i.v. zu verabreichenden Standardimmunglobulins innerhalb von 2-3 Tagen nach Kontakt lässt sich mit großer Wahrscheinlichkeit vermeiden, dass diese Patienten an Masern erkranken, Bei späterer Gabe bis zum 6. Tag ist zumindest noch die Mitigierung der Krankheit möglich (www.rki.de/DE/Content/Infekt/EpidBull/Archiv/2017/ Ausgaben/02_17.pdf?_blob=publicationFile).

Dabei sollte Folgendes beachtet werden: Nach einer Immunglobulin-Gabe ist die MMR-Impfung für 5-6 Monate nicht sicher wirksam.

\section{Versehentliche (akzidentelle) Masernimpfung bei (vorher nicht bekannten) Immundefekten}

\section{Zelluläre Immundefekte}

Bei akzidenteller Masernimpfung von Kindern mit schweren zellulären Immundefekten (z. B. SCID) kann es zu schwerwiegenden und letalen Komplikationen kommen (z. B. Riesenzell-Pneumonie, „measles inclusion-body encephalitis“ (MIBE) [2, 17, 21].

Bei partiellem DiGeorge-Syndrom (Mikrodeletion 22q11.21, T-Zellen sind nur leicht erniedrigt, siehe auch Artikel von S. Ehl und N. Wagner in diesem Heft) dagegen ist die Masernimpfung meist gut verträglich und wirksam $[13,22]$.

\section{Defizienzen in der angeborenen antiviralen Immunität (Interferon-Signalweg) (20)}

Bei sehr wenigen Patienten mit spezifischen Störungen der angeborenen antiviralen Immunität traten nach MMR-Impfung schwere neurologische und z. T, tödliche Komplikationen auf. Bei diesen Patienten konnten Mutationen im Interferon-Signalweg in den Genen IRF3, IRF7, IRF9, STAT1, STAT2, IFNAR1 und IFNAR2 nachgewiesen werden [11]. Möglicherweise gibt es darüber hinaus noch weitere entsprechende Prädispositionsfaktoren. Die sehr wenigen betroffenen Patienten sollten unbedingt molekulargenetisch untersucht werden (u.a. Trio-Exom-Sequenzierung, Interferon-Signatur). Sollte sich dabei eine krankheitsverursachende Mutation finden, sollten - möglichst noch vor einer Masernimpfung - auch kleine Geschwister diesbezüglich untersucht werden. Im positiven Fall dürfen diesbezüglich gefährdete Kinder selektiv nicht gegen Masern geimpft werden. Sie erhalten bei Masernkontakt schnellstmöglich Immunglobuline.

\section{Reine B-Zell-Defekte (z. B. Bruton Agammaglobulinämie)}

Betroffene Kinder zeigen keine vermehrte Empfänglichkeit gegenüber Maserninfektionen, da die zelluläre Immunität intakt ist. Sie erhalten frühzeitig regelmäßig Immunglobuline (subkutan oder intravenös), eine Masernimpfung ist daher nicht mehr sinnvoll (Neutralisierung durch Immunglobuline).

\section{Masernimpfung bei erworbener Immunsuppression}

In einem Review von 2015 [8] zeigten Kinder mit juveniler idiopathischer Arthritis (JIA) unter Therapie mit niedrig dosiertem Methotrexat (10-15 mg/m²), TNF $\alpha$ - sowie IL1R-Antagonisten nach einer MMR-Booster-Impfung keine Verschlechterung der klinischen Symptomatik. Die Impfung war dabei ausreichend immunogen [10]. Insgesamt ist die Datenlage bei pädiatrischen Patienten noch dürftig. In diesem Zusammenhang verweisen wir auf den Artikel von Ehl und Wagner: „Impfen bei Primären Immundefekten, Autoimmunkrankheiten und anderen chronisch entzündlichen Erkrankungen unter immunmodulatorischer Therapie“, in diesem Heft.

\section{Epidemiologische Besonderheiten von Masern in Europa und in Deutschland}

Aus verschiedenen Gründen steigt in einigen Europäischen Ländern - trotz Impfprogrammen - die Zahl der Masern-Erkrankungen z. T. stark an (v. a. seit 2017). Der größte Anstieg der Erkrankungszahlen findet sich in der Ukraine (dunkelbraun), in Kasachstan (rot) und in Georgien (dunkelblau). In allen Fällen ist dies auf die niedrige Impfbereitschaft bzw. Probleme im Gesundheitssystem (u. a. Impfstoffmangel) zurückzuführen ( $\triangleright$ Abb.2). 


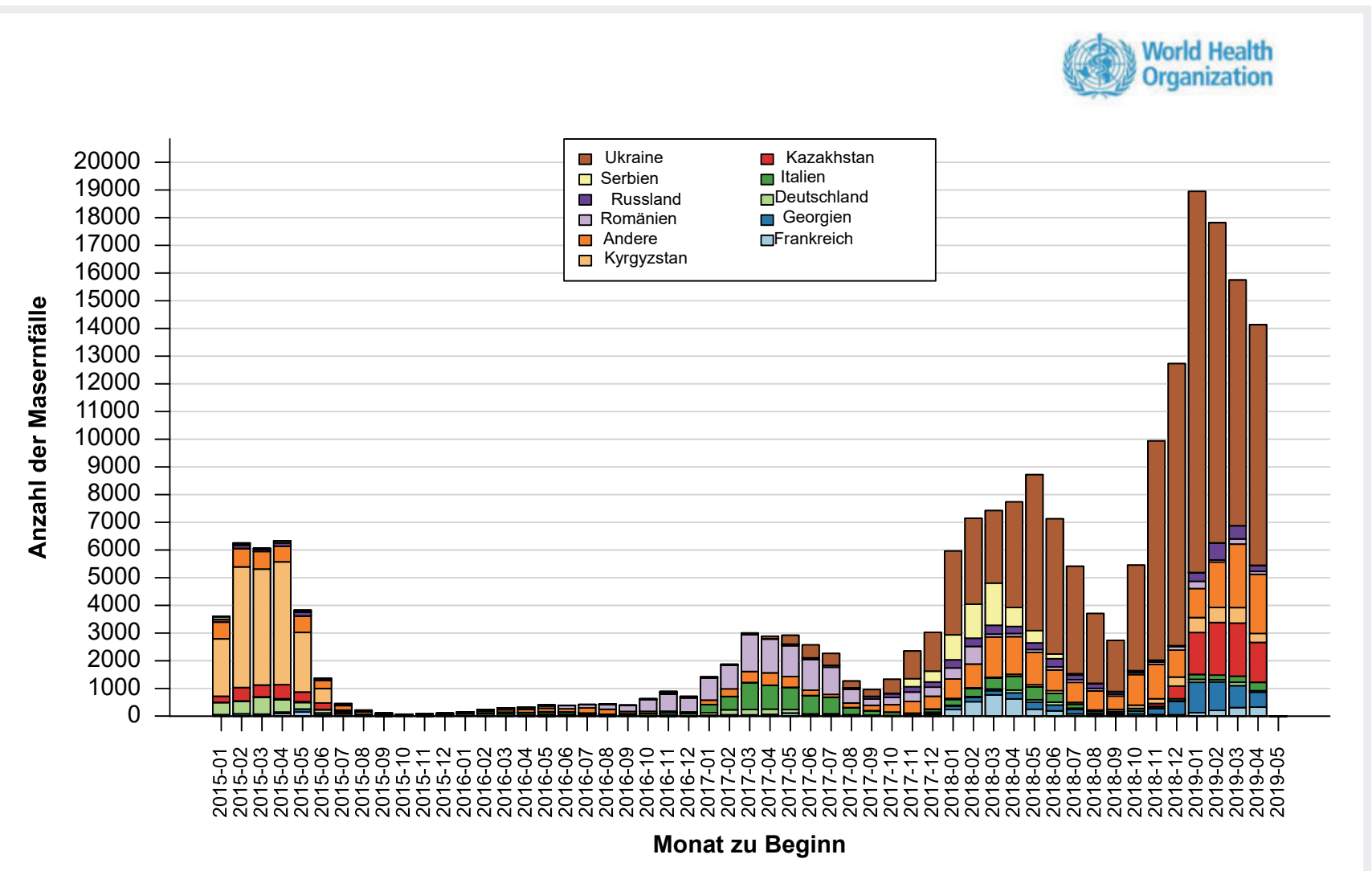

- Abb. 2 Masernerkrankungen in Europa (2015 bis Mai 2019). Der größte Anstieg der Erkrankungszahlen findet sich aktuell in der Ukraine (dunkelbraun), in Kasachstan (rot) und in Georgien (dunkelblau). In den meisten Fällen ist dies auf die niedrige Impfbereitschaft bzw. Probleme im Gesundheitssystem (u. a. Impfstoffmangel) zurückzuführen (ID: 285428 Permission authorization for WHO copyrighted material, E-Mail vom 25.4.2019).

Die WHO registrierte in den ersten 6 Monaten im Jahr 2019 in 48 europäischen Ländern sogar mehr als doppelt so viele Masernfälle (89 994) als im Vorjahresvergleichszeitraum (44 175). 2018 starben in der Europäischen Region 72 Kinder und Erwachsene an Masern, in den ersten 6 Monaten 2019 waren es 37 Todesfälle (Spiegel online v. 29.08.2019; http://www.euro.who.int/de/media-centre/sections/ press-releases/2019/measles-in-europe-record-numberof-both-sick-and-immunized).

\section{Wie sieht es bezüglich des Masern- Impfverhaltens in Deutschland aus?}

Bei der 1. Masern-Impfung bei Kindern im Alter von 15 Monaten lag die Durchimpfungsquote in Deutschland insgesamt nur bei $87,3 \%$ (im Alter von 36 Monaten bei $98 \%$; Geburtsjahrgang 2013), bei der 2. Impfung im Alter von 36 Monaten bei 86,1 \% (Geburtsjahrgang 2013) (RKI).

Nach einer aktuellen Studie durch die Barmer Krankenkasse lag die Masern-Durchimpfungsquote hier nach 2 Jahren sogar nur bei 78, $9 \%$ (2015), nach 4 Jahren bei $87,4 \%$ (2013) und nach 6 Jahren bei nur 87,4\% (2013), also weiterhin deutlich unter den von der WHO angestrebten $95 \%$
Durchimpfungsrate (Arzneimittelreport 2019. Impfungen bei Kindern und Jugendlichen, 2019).

Aufgrund der niedrigen Durchimpfungsquoten kommt es seit vielen Jahren in Deutschland immer wieder zu Masernausbrüchen mit schweren Komplikationen und Todesfällen (bis maximal zu 2383 Erkrankungen im Jahr 2015,

- Abb. 1). Der untere Teil von Abbildung 1 zeigt, dass überwiegend (nicht geimpfte) Erwachsene an Masern erkranken (braune Balken, Altersgruppe 15-40+). Dies zeigt, dass diese Altersgruppe mehr in das „Masern-Impfprogramm“ einbezogen werden muss.

Auch regulär 2-mal geimpfte Personen (bei denen die Impfung 2-mal nicht „angegangen“ ist, können in einem geringen Prozentsatz an Masern erkranken (grün). Die rechtsseitige Tabelle ( $\triangleright$ Abb. $\mathbf{1}$ ) zeigt, dass in den letzten Jahren bis über 2000 Personen/Jahr (gilt für das Jahr 2015) an Masern erkrankt sind. Der obere Teil der Abbildung zeigt die schwankende Masern-Inzidenz in Deutschland seit 2017. 
Merke: Bei einer Masern-Impfquote mit 2 Impfungen von jeweils $\geq 95 \%$ ist es generell möglich, eine endemische Virusübertragung in einer definierten Region zu unterbrechen (WHO).

Das Ziel der Elimination der Masern und Röteln in der Europäischen WHO-Region sollte ursprünglich bis Ende 2015 erreicht werden. Auch Deutschland hat sich wiederholt zu diesen Zielen bekannt. Im »Interventionsprogramm Masern, Mumps, Röteln (MMR)« des Bundes und der Länder aus dem Jahr 1999 wurden erstmals Leitziele für eine Elimination der Masern festgelegt. Das Programm sah langfristig eine Senkung der Maserninzidenz auf < 1 Fall pro 100000 Einwohner pro Jahr durch konsequente Impfungen mit Kombinationsimpfstoffen gemäß STIKO-Empfehlungen vor. Der Nationale Impfplan, der von den Ländern und vom Bund sowie von weiteren im Impfwesen Verantwortlichen Anfang 2012 veröffentlicht wurde, unterstreicht insbesondere das WHO-Ziel der Masern- und Röteln-Elimination bis 2015 und regt eine Aktualisierung des deutschen Interventionsprogramms an. Mittlerweile wurde das Ziel der Elimination der Masern und Röteln in Deutschland auf 2020 verschoben (Nationaler Aktionsplan 2015-2020).

Eine Masern-Impfpflicht besteht derzeit in 10 Europäischen Ländern (Frankreich, Italien, Lettland, Tschechien, Slowakei, Slowenien, Kroatien und Bulgarien, Polen und Ungarn). Dieses Vorgehen ist allerdings nicht zwingend ein Erfolgsrezept. So haben die Niederlande die Masern auch ohne Impfpflicht weitgehend eliminiert (Ausnahme: Masernausbrüche im „Bibelgürtel“), während andere Länder trotz Impfpflicht noch gegen Masern kämpfen (Quelle: ZDFcheck19, Mai 2019).

Am 14.11.2019 hat der Deutsche Bundestag eine Impfpflicht für Masern für Deutschland beschlossen. Sie gilt unter anderem in Kitas, Schulen und Flüchtlingsunterkünften. Verweigerern drohen Bußgelder. Die Regelung trat am 01.03.2020 in Kraft. Der deutsche Ethikrat empfiehlt aus verschiedenen Gründen eine Impfpflicht nur für Berufsgruppen in besonderer Verantwortung (Personal im Gesundheits-, Sozial- und Bildungswesen), nicht hingegen für alle Erwachsenen oder Kinder (https://www.ethikrat. org/fileadmin/Publikationen/Stellungnahmen/deutsch/ stellungnahme-impfen-als-pflicht.pdf.) Erst in den nächsten Jahren wird sich zeigen, ob die Masern-Impfstrategie letztlich erfolgreich war.

\section{FAZIT FÜR DIE PRAXIS}

Das Nutzen-Risikoprofil der Masern-Lebendimpfung (meist MMR oder MMRV) ist hervorragend. Schwere Nebenwirkungen sind extrem selten $(<1000000)$. Auch bei Hühnereiweißallergie kann mit Masernlebendimpfstoff (z. B. MMR) geimpft werden (Ausnahme: anamnestisch frühere Anaphylaxie nach Hühnerweißverzehr).

Um eine ausreichende Herdimmunität zu erreichen, ist eine Masern-Durchimpfungsquote von mind. 95\% anzustreben. Ob dies nur durch eine Impfpflicht (seit 01.03.2020) zu erreichen ist, bleibt abzuwarten. Säuglinge von Müttern, die früher Wildmasern durchgemacht haben, haben einen längeren Immunschutz als Säuglinge von Masern-geimpften Müttern.

Die Masernimpfung darf nicht bei schweren Immundefekten (z. B. SCID) und bekannten Störungen im Interferon-Signalweg gegeben werden.

In Ländern mit einer konsequenten Impfpolitik und somit einem drastischen Rückgang der natürlichen Masern ist die SSPE fast verschwunden.

\section{Wissenschaftlich verantwortlich}

Wissenschaftlich verantwortlich gemäß Zertifizierungsbedingungen für diesen Beitrag ist Prof. Dr. med. Volker Schuster.

\section{Interessenkonflikt}

\section{Erklärung zu finanziellen Interessen}

Forschungsförderung erhalten: ja; Honorar/geldwerten Vorteil für Referententätigkeit erhalten: ja; Bezahlter Berater/interner Schulungsreferent/Gehaltsempfänger: ja; Patent/Geschäftsanteile/Aktien (Autor/Partner, Ehepartner, Kinder) an Firma: nein.

Erklärung zu nicht finanziellen Interessen

Der Autor gibt an, dass kein Interessenkonflikt besteht.

\section{Korrespondenzadresse}

Prof. Dr. med. Volker Schuster

Universitätsklinik und Poliklinik für Kinder und Jugendliche Leipzig

Liebigstraße 20a, 04103 Leipzig

Tel.: 0341 97-26242, Fax: -26229

E-Mail: volker.schuster@medizin.uni-leipzig.de 
Literatur

[1] Bester JC. Measles and Measles Vaccination: A Review. JAMA Pediatr 2016; 170 (12): 1209-1215

[2] Bitnun A, Shannon P, Durward A et al. Measles inclusionbody encephalitis caused by the vaccine strain of measles virus. Clin Infect Dis 1999; 29 (4): 855-861

[3] Bolotin S, Hughes SL, Gul N et al. What Is the Evidence to Support a Correlate of Protection for Measles? A Systematic Review. J Infect Dis 2019 Nov 1. pii: jiz380. doi: 10.1093/ infdis/jiz380

[4] Borte S, Liebert UG, Borte M et al. Efficacy of measles, mumps and rubella revaccination in children with juvenile idiopathic arthritis treated with methotrexate and etanercept. Rheumatology (Oxford) 2009; 48 (2): 144-148

[5] Clark AT, Skypala I, Leech SC et al. British Society for Allergy and Clinical Immunology guidelines for the management of egg allergy. Clin Exp Allergy 2010; 40 (8): 1116-1129

[6] Fiebelkorn AP, Coleman LA, Belongia EA et al. Measles Virus Neutralizing Antibody Response, Cell-Mediated Immunity, and Immunoglobulin G Antibody Avidity Before and After Receipt of a Third Dose of Measles, Mumps, and Rubella Vaccine in Young Adults. J Infect Dis 2016; 213 (7): 1115-1123

[7] Garg RK, Mahadevan A, Malhotra HS et al. Subacute sclerosing panencephalitis. Rev Med Virol 2019; 29 (5): e2058

[8] Groot N, Heijstek MW, Wulffraat NM. Vaccinations in paediatric rheumatology: an update on current developments. Curr Rheumatol Rep 2015; 17 (7): 46

[9] Guerra FM, Crowcroft NS, Friedman L et al. Immunity of Canadians and Risk of Epidemics (iCARE) Network. Waning of measles maternal antibody in infants in measles elimination settings - A systematic literature review. Vaccine 2018; 36 (10): 1248-1255

[10] Heijstek MW, Kamphuis S, Armbrust W et al. Effects of the live attenuated measles-mumps-rubella booster vaccination on disease activity in patients with juvenile idiopathic arthritis: a randomized trial. JAMA 2013; 309 (23): 2449-2456

[11] Hernandez N, Bucciol G, Moens L et al. Inherited IFNAR1 deficiency in otherwise healthy patients with adverse reaction to measles and yellow fever live vaccines. J Exp Med 2019; 216 (9): 2057-2070

[12] Hviid A, Hansen JV, Frisch M et al. Measles, Mumps, Rubella Vaccination and Autism: A Nationwide Cohort Study. Ann Intern Med 2019; 170 (8): 513-520

[13] Hofstetter AM, Jakob K, Klein NP et al. Live vaccine use and safety in DiGeorge syndrome. Pediatrics 2014; 133 (4): e946-954

[14] James JM, Burks AW, Roberson PK et al. Safe administration of the measles vaccine to children allergic to eggs. $N$ Engl J Med 1995; 332: 1262-1266

[15] Jeyaratnam J, Ter Haar NM, Lachmann HJ et al. The safety of live-attenuated vaccines in patients using IL- 1 or IL- 6 blockade: an international survey. Pediatr Rheumatol Online J 2018; 16 (1): 19

[16] Knuf M, Zepp F, Meyer CU et al. Safety, immunogenicity and immediate pain of intramuscular versus subcutaneous administration of a measles-mumps-rubella-varicella vaccine to children aged 11-21 months. Eur J Pediatr 2010; 169: 925-933

[17] Mihatsch MJ, Ohnacker $H$, Just $M$ et al. Lethal measles giant cell pneumonia after live measles vaccination in a case of thymic alymphoplasia Gitlin. Helv Paediatr Acta 1972; 27 (2): $143-146$
[18] Mina M], Metcalf C], de Swart RL et al. Long-term measlesinduced immunomodulation increases overall childhood infectious disease mortality. Science 2015; 348 (6235): 694-699

[19] Mina MJ, Kula T, Leng Y et al. Measles virus infection diminishes preexisting antibodies that offer protection from other pathogens. Science 2019; 366 (6465): 599-606

[20] Mogensen TH. IRF and STAT Transcription Factors - From Basic Biology to Roles in Infection, Protective Immunity, and Primary Immunodeficiencies. Front Immunol 2019; 9: 3047

[21] Monafo W], Haslam DB, Roberts RL et al. Disseminated measles infection after vaccination in a child with a congenital immunodeficiency. J Pediatr 1994; 124 (2): 273-276

[22] Perez EE, Bokszczanin A, McDonald-McGinn D et al. Safety of live viral vaccines in patients with chromosome 22q11.2 deletion syndrome (DiGeorge syndrome/velocardiofacial syndrome). Pediatrics 2003; 112 (4): e325

[23] Petrova VN, Sawatsky B, Han AX et al. Incomplete genetic reconstitution of $B$ cell pools contributes to prolonged immunosuppression after measles. Sci Immunol 2019; 4 (41)

[24] Laksono BM, de Vries RD, Verburgh RJ et al. Studies into the mechanism of measles-associated immune suppression during a measles outbreak in the Netherlands. Nat Commun 2018; 23; 9 (1): 4944

[25] Schönberger K, Ludwig MS, Wildner M et al. Epidemiology of subacute sclerosing panencephalitis (SSPE) in Germany from 2003 to 2009: a risk estimation. PLoS One 2013; 8 (7): e68909

[26] Science M, Savage R, Severini A et al. Measles Antibody Levels in Young Infants. Pediatrics 2019; 144 (6): 20190630

[27] Strebel PM, Orenstein WA. Measles. N Engl J Med.2019; 381 (4): 349

[28] Taylor LE, Swerdfeger AL, Eslick GD. Vaccines are not associated with autism: an evidence-based meta-analysis of case-control and cohort studies. Vaccine 2014; 32: 3623-3629

[29] Wakefield A], Murch SH, Anthony A et al. Ileal-lymphoidnodular hyperplasia, non-specific colitis, and pervasive developmental disorder in children. Lancet 1998; 351 (9103): 637-641. Retraction in: Lancet 2010; 375 (9713): 445

[30] Weissbrich B. Masern-Infektion, Folgeerkrankungen und Masern-Impfung Bayerisches Ärzteblatt 2015; 5: 240-242

[31] White SJ, Boldt KL, Holditch S] et al. Measles, mumps, and rubella. Clin Obstet Gynecol 2012; 55 (2): 550-559

Bibliografie

DOI https://doi.org/10.1055/a-1113-3316

Kinder- und Jugendmedizin 2020; 20: 83-91

(c) Georg Thieme Verlag KG Stuttgart · New York ISSN 1617-0288 


\section{Punkte sammeln auf CM/F.thieme.de}

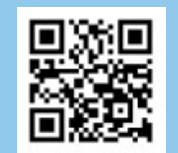

Diese Fortbildungseinheit ist bis zu 12 Monate online für die Teilnahme verfügbar.

Den genauen Einsendeschluss finden Sie beim Modul auf https://cme.thieme.de/CXELAXK.

Sollten Sie Fragen zur Online-Teilnahme haben, finden Sie unter https://cme.thieme.de/hilfe

eine ausführliche Anleitung. Wir wünschen viel Erfolg beim Beantworten

der Fragen!

Unter https://eref.thieme.de/CXELAXK oder über den QR-Code kommen Sie

direkt zum Artikel.

VNR 2760512020158724332

\section{Frage 1}

Welche unerwünschte Wirkung kann nach der MMR-Impfung auftreten?

A subakute sklerosierende Panenzephalitis (SSPE)

B Fieberkrämpfe

C Autismus

D sekundäre Immunsuppression

E Verbreitung des Masern-Virus durch den Impfling

\section{Frage 2}

Für die MMR-Impfung gilt:

A Eine niedrigdosierte Therapie mit Methotrexat (10-15 mg/ $\mathrm{m}^{2}$ ) stellt immer eine klare Kontraindikation für eine MMRImpfung dar.

B Bei einer Agammaglobulinämie (kompletter Antikörpermangel) ist die MMR-Impfung nicht sinnvoll.

C Impfmasern sind hoch ansteckend.

D Die MMR-Impfung darf nicht bei Hühnereiweißallergie verabreicht werden.

E Die MMR-Impfung ist auch bei Schwangeren indiziert.

\section{Frage 3}

Für die MMR-Impfung gilt:

A Die MMR-Impfung sollte niemals zusammen mit Totimpfstoffen (z. B. 6-Fachimpfstoff DTaP-IPV-HB-Hib, Pneumokokkenund Meningokokken-Impfstoffe) verabreicht werden.

B Eine Hühnereiweißallergie ist normalerweise keine Kontraindikation für eine MMR-Impfung.

C Die STIKO empfiehlt die erste MMR-Impfung im Alter von 4 Jahren.

D Die MMR-Impfung schützt auch vor Varizellen.

E Die Letalität bei der MMR-Impfung liegt bei 1:5000.

\section{Frage 4}

Welche Angabe ist richtig?

A Eine MMR-Impfpflicht wird es in Deutschland niemals geben.

B Die SSPE kann durch Impfmasern ausgelöst werden.

C Bei MMR-Durchimpfungsraten von $>55 \%$ ist eine Eradikation von Masern bereits nach 5 Jahren möglich.

D Wildmasern führen zu einer ausgeprägten Immunsuppression über Monate bis Jahre.

E Masern sind wenig ansteckend.

\section{Frage 5}

Welche Antwort ist richtig?

A Die Masern-Postexpositionsprophylaxe bedeutet u.a., dass die betroffene Person für mind. 10 Tage in Quarantäne kommt.

B Die versehentliche MMR-Impfung von Kindern mit schwerem zellulärem Immundefekt kann zu schweren, ggfs auch tödlichen Komplikationen führen.

C Der Zusammenhang zwischen Masern-Impfung und Autismus ist mittlerweile klar bewiesen.

D Sog. „Masernpartys“ sind vollkommen ungefährlich.

E Die Masern-Epidemiologie in Deutschland hat klar gezeigt, dass 2x MMR-geimpfte Personen überhaupt nicht mehr an Masern erkranken können.

\section{Frage 6}

Welche Antwort ist richtig?

A Der mütterlich übertragene Masernschutz bei jungen Säuglingen ist bei MMR-geimpften Frauen höher als bei Frauen, die früher Wildmasern durchgemacht haben.

B Bei versehentlicher MMR-Impfung in der Schwangerschaft muss sofort eine Interruptio eingeleitet werden.

C Das Fieberkrampfrisiko durch die 1. MMRV-Impfung ist im Vergleich zur getrennt verabreichten 1. Impfung mit MMR und Varizellen erhöht.

D Die STIKO empfiehlt allen Frauen, die nach 1945 geboren sind und nicht bzw. in der Kindheit nur einmal gegen Masern geimpft wurden, eine einmalige Impfung gegen Masern.

E Die MMR- und MMRV-Impfung wird streng intrakutan appliziert.

\section{Frage 7}

Was trifft zu?

A Masern kommen ubiquitär vor.

B Die Masern-Enzephalitis hat eine gute Prognose.

C Die MMR-Impfung ist mit einer Letalität von $5 \%$ assoziiert.

D Die MMR-Impfung führt in seltenen Fällen zu einer SSPE.

E Die WHO strebt eine Masern-Durchimpfungsrate von $100 \%$ an.

- Weitere Fragen auf der folgenden Seite ... 


\section{Punkte sammeln auf CME.thieme.de}

Fortsetzung ...

\section{Frage 8}

Welche Antwort ist richtig?

A Der größte Anstieg der Masern-Erkrankungszahlen in Europa (Zeitraum 2015 bis Mai 2019) findet sich in Deutschland.

B In Deutschland wurde das Ziel der Elimination der Masern und Röteln zuletzt auf 2020 verschoben.

C Bei den Masernausbrüchen in Deutschland ist am stärksten die Altersgruppe der 5-9 Jahre alten Kinder betroffen.

D Die meisten der an Masern erkrankten Personen waren einmalig gegen Masern geimpft.

E WHO-Ziel ist die Senkung der Maserninzidenz auf ca. 10 Fälle pro 100000 Einwohner pro Jahr.

\section{Frage 9}

Welche Antwort ist richtig?

A Nach durchgemachten Masern hält der „natürliche“ Immunschutz höchstens 5 Jahre lang an.

B In Deutschland gibt es derzeit nur einen monovalenten Masernimpfstoff.

C Zu den Nebenwirkungen einer MMR-Impfung gehören auch Otitis media und Gastroenteritis.

D Nach Masernkontakt eines nicht geschützten (gefährdeten) Kindes kann die frühzeitige Gabe von Standardimmunglobulin den Ausbruch von Masern mit großer Wahrscheinlichkeit verhindern.

E MMR-geimpfte Kinder entwickeln gehäuft Autismus.

\section{Frage 10}

Welche Antwort ist richtig?

A Eine Thrombozytopenie ist eine seltene Komplikation nach MMR-Impfung.

B Hartnäckigen MMR-Impfungs-Verweigerern drohen nach Einführung der Impfpflicht 2020 Gefängnisstrafen.

C Die MMR-Impfung führt bei Hühnereiweißallergie häufig zu einer Anaphylaxie.

D Nach STIKO-Empfehlung sollen ab 2020 alle Kinder 3-mal gegen Masern geimpft werden.

E Schwangere sollten im letzten Trimenon nochmals MMR geimpft werden, um die Masern-Immunität des Neugeborenen und Säuglings zu verbessern. 